\title{
Characterization of Novel Ceramic Composite Nanofibers by Electron Microscopy
}

Matthew T. Janish ${ }^{1}$, Fei Huang ${ }^{2}$, Lichun Zhang ${ }^{3}$, Vahid Rastegar ${ }^{1}$, Nathan Martin ${ }^{1}$, Jason Chan ${ }^{1}$, Bonnie B. McKenzie ${ }^{4}$, Joseph R. Michael ${ }^{4}$, Chris Cornelius², and C. Barry Carter ${ }^{5}$

${ }^{1}$ Department of Materials Science and Engineering, University of Connecticut

${ }^{2}$ Department of Chemical and Biomolecular Engineering, University of Connecticut

${ }^{3}$ Institute of Materials Science, University of Connecticut

${ }^{4}$ Sandia National Laboratories, Albuquerque, NM 87185-1405

${ }^{5}$ Department of Materials, Chemical, and Biomolecular Engineering, University of Connecticut

While the process of electrospinning fibers has been known for over a century[1], it was the rise of nano-materials in the '90s that transformed it from an obscure technique to one that is both academically and commercially interesting. Compared to other nanofiber fabrication processes, electrospinning has the advantage of being cheap and relatively high volume. Its resurgence was initially directed towards polymer nanofiber membranes, but as the process has matured so has its range of applicability broadened to include both metal [2] and ceramic fibers. Here we describe the characterization of $\mathrm{TiO}_{2} / \mathrm{SiO}_{2}$ composite nanofibers decorated with donut-shaped nanoparticles (NPs). The fibers are being produced for use as membranes in energy production and storage applications.

A field-emission scanning electron microscope (FESEM) was used for preliminary analysis and to determine whether the specimens were suitable for examination in a transmission electron microscope (TEM). The SEM secondary electron images revealed some fibers that were smooth and some decorated with NPs, with fiber diameters ranging from $100 \mathrm{~nm}$ to $10 \mu \mathrm{m}$. The fibers decorated with NPs were not from the same batch as the fibers without NPs, indicating that the presence of the NPs may be controlled by the process. Fiber composition is also controllable, with Ti:Si ratios of 1:3, 1:1, 3:1, and 9:1 being verified by X-ray energy dispersive spectrometry (XEDS).

A SEM/focused ion beam (FIB) was used both to examine fiber and NP cross-sections and to prepare cross-section specimens for the TEM. After milling away the conductive surface of a NP-decorated fiber it was discovered that the core of the fibers are highly insulating, as the image became distorted due to charging effects. This suggests a core-shell morphology wherein an insulating core is surrounded by a conductive outer layer. The particles on the fiber did not share this characteristic; rather they were conductive and homogeneous throughout.

Two methods were used to produce TEM specimens from the as-prepared fibers. In the first method the fibers were spun directly onto a 3mm copper TEM grid. In the second method the fibers were dispersed in ethanol using a sonicator. A small capillary tube was used to place a single drop of this suspension on the TEM grid. When the grid had fully dried, SEM analysis revealed several individual fibers laying across holes in the grid, and XEDS confirmed that they were chemically unaltered.

The preliminary TEM analysis will be reported.

[1] S. Ramakrishna et al., Materials Today 9, 3 (2006) 40-50.

[2] H. Wu et al., Nano Letters 10 (2010) 4242-4248.

[3] C.B. Carter and M.G. Norton, Ceramic Materials: Science and Engineering, $2^{\text {nd }}$ ed., Springer 2013. 
[4] M.T.J. would like to acknowledge the Institute of Materials Science at the University of Connecticut for access to the JEOL 6335F FESEM and the FEI Strata 400S DualBeam FESEM/FIB, and the Center for Integrated Nanotechnologies at the Sandia National Labs for access to their equipment. Funding for this project was provided by the Department of Education's GAANN fellowship program. B.B.K. and J.R.M. are supported by CINT. Sandia National Laboratories is a multi-program laboratory managed and operated by Sandia Corporation, a wholly owned subsidiary of Lockheed Martin Corporation, for the U.S. Department of Energy's National Nuclear Security Administration under contract DE-AC0494AL85000.

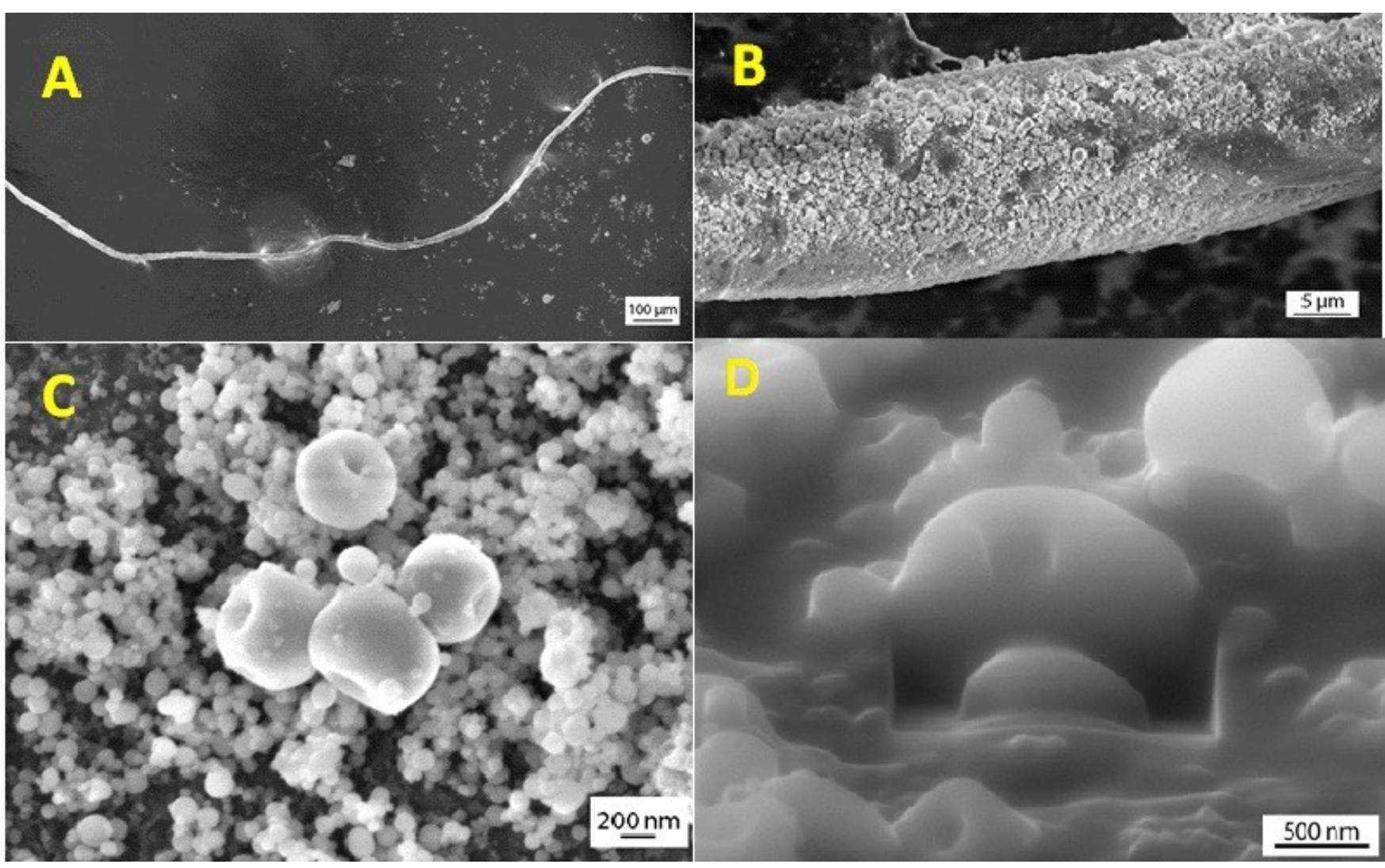

Fig.1 A single electrospun fiber at different magnifications $(A, B)$ together with particles that decorate the fiber $(\mathrm{C})$ and a FIB image of a particle cross-section (D).
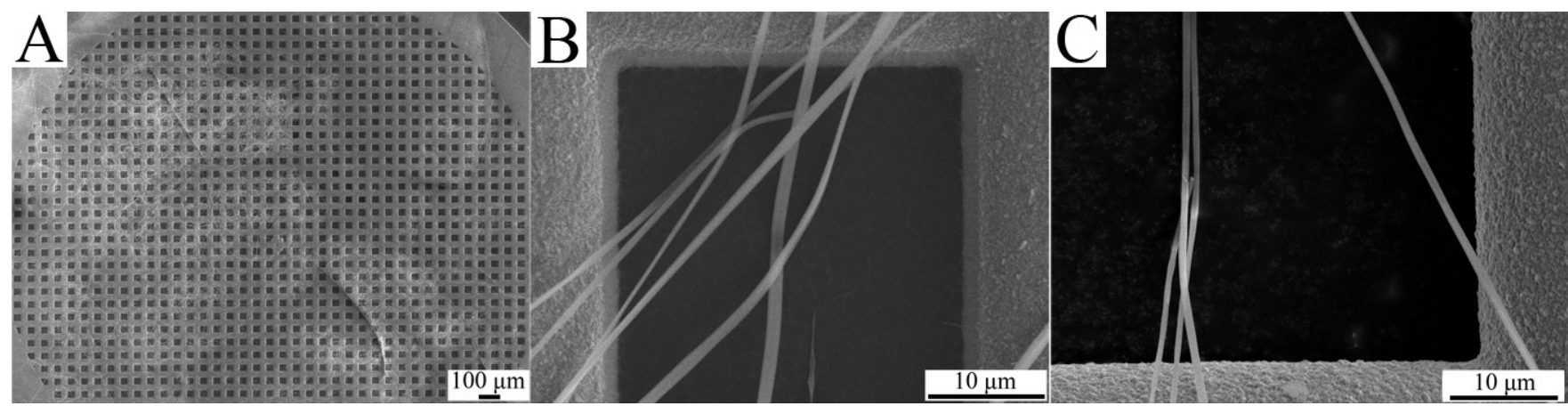

Fig.2 Electrospun fibers that have been spun onto a copper TEM grid (A,B) and deposited onto a grid after being dispersed in ethanol (C). 